\title{
Calculation of Positron Response from Embedded Nanoparticles
}

\author{
J. KuRIPLACH \\ Department of Low Temperature Physics, Charles University \\ V Holešovičkách 2, 18000 Prague, Czech Republic
}

\begin{abstract}
Nanoparticles embedded in a matrix can trap positrons under certain conditions. In such cases nanoparticles can be effectively studied by means of positron annihilation because positron annihilation characteristics contain information related to nanoparticles' electronic and atomic structure. Of great importance is to calculate the positron response from such nanoparticles. Then, nanoparticles can, in principle, be identified by comparing the measured and calculated positron annihilation response. For this purpose we present an efficient computational method based on the well-known atomic superposition technique. This method is explained in detail, justified on the basis of first principles calculations, and applied to $\mathrm{Cu}$ nanoparticles embedded in the Fe matrix.
\end{abstract}

PACS numbers: 78.70.Bj, 71.60.+z, 61.46.+w, 81.40.Np

\section{Introduction}

The investigation of nanostructures becomes increasingly important due to both scientific and practical aspects. The precise microstructure characterization is a necessary prerequisite for the understanding of various properties of nanostructures and positron annihilation (PA) spectroscopy may certainly bring unique details about the microstructure of nanostructured materials.

Nanosized particles are formed in many age-hardening systems and can be also created by ion implantation and subsequent annealing. Provided that the positron affinity of such particles is lower than that of the matrix, positrons may get trapped in them. This effect was first observed experimentally about 25 years ago [1] and described from the theoretical point of view by several authors $[2,3]$, but until recently $[4,5]$ an effective computational method to determine the positron response from nanoparticles was missing. 
In general, ab initio methods can be used for such studies, but the number of atoms included in the studied cell may hardly exceed a few hundreds and only very small particles can be studied. On the other hand, the so-called atomic superposition (ATSUP) method [6] is routinely used in positron calculations for cells containing thousands of atoms. The ATSUP method, however, neglects charge transfer effects that are important for the alignment of Fermi levels of the host and embedded particles [3]. As explained in [5], this problem can be circumvented by creating an appropriate potential well for positrons at the particles. This model approach requires only two parameters which need to be found from moderate size $a b$ initio calculations. For completeness, we mention that there have been already attempts to study PA at nanoparticles using the ATSUP method (see, e.g., [7]), but to our knowledge the effect of the positron affinity has been always neglected.

In this work we apply this method to the $\mathrm{Fe}-\mathrm{Cu}$ system where $\mathrm{Cu}$ precipitates are well known to trap positrons [8]. In particular, we find values of the above-mentioned parameters and calculate the dependence of the so-called wing parameter of Doppler broadening spectra on the size of $\mathrm{Cu}$ particles embedded in the $\mathrm{Fe}$ matrix. The $\mathrm{Fe}-\mathrm{Cu}$ system serves as a model alloy for reactor pressure vessel steels where $\mathrm{Cu}$-rich precipitates play the key role in the process of embrittlement [9].

\section{Computational method}

The method [5] introduced recently for calculations of positron characteristics of embedded nanoparticles consists of three steps. We repeat them briefly here. In the first step the energy $\left(E_{1}\right)$ of positrons in the defect-free bulk material is found. Second, the positron potential in the vicinity of atoms constituting the particle is shifted (by $\Delta V_{2}$ ) in order to have the positron energy equal to $E_{1}$. In this way a reference energy level for the positron potential of atoms constituting the particle is found*. This corresponds to the hypothetical case when the positron affinity difference $\left(\Delta A_{+}\right)$of the host and particle is zero and the positron is delocalized both in the host and particle. Third, the positron potential is further shifted by the energy corresponding to $\Delta A_{+}$(i.e. the resulting shift $\Delta V_{3}=\Delta V_{2}-\Delta A_{+}$) and, thereby, the potential well with the correct depth is created.

Now we need to find the values of parameters of this model. First, positron affinities $\left(A_{+}\right)$of $\mathrm{Fe}$ and bcc $\mathrm{Cu}$ were calculated using the linear-muffin-tin-orbital (LMTO) method [10]. The results are as follows: $A_{+}(\mathrm{Fe})=-3.8 \mathrm{eV}$ and $A_{+}(\mathrm{Cu})=-4.5 \mathrm{eV}$. These values agree reasonably well with those calculated in [3] and with experiment [11]. Thus, the resulting difference of positron affinities of $\mathrm{Fe}$ and $\mathrm{Cu}$ is $0.7 \mathrm{eV}\left(=\Delta A_{+}\right)$. This is the first parameter of our model. We note that the value of the positron affinity for fcc $\mathrm{Cu}$ is only by $0.1 \mathrm{eV}$ larger than that

\footnotetext{
*It should be noted that in supercells used for calculations the number of atoms in the particle must be much lower than that of the matrix.
} 
for bcc $\mathrm{Cu}$ (with the same lattice constant as $\mathrm{Fe}$ ). Positron affinity calculations were carried out using the gradient-correction (GC) scheme for electron-positron correlations by Barbiellini et al. [12].

Second, the potential shift is applied in spheres centered at sites of nuclei of atoms. The radius $(R)$ of such spheres is the next parameter of our model. In fact, this parameter controls the size of the potential well created. In order to find the value of $R$, we employed the Vienna ab initio simulation package (VASP) developed at the Institute für Materialphysik of the Universität Wien [13]. We made use of the projected augmented wave (PAW) scheme [14] and PAW potentials supplied with the VASP. We calculated the electronic structure of small $\mathrm{Cu}$ particles in Fe. In particular, we used 54 and 128 atom based supercells $(3 \times 3 \times 3$ and $4 \times 4 \times 4 \mathrm{Fe}$ bcc cells, respectively) with $\mathrm{Cu}$ particles located in their centers. No lattice relaxations were considered. This can be justified by taking into account that the atomic volumes for $\mathrm{Cu}$ and $\mathrm{Fe}$ atoms are very similar and $\mathrm{Cu}$ precipitates in Fe are known to be coherent with the matrix up to about $4 \mathrm{~nm}$ size (see [8] and references therein). From various possibilities how to create $\mathrm{Cu}$ particles in the bcc lattice we considered the most compact atomic configurations.

The valence electron density and Coulomb potential produced by the VASP are read by the ATSUP program where the core electron density and Coulomb potential are added. Then, the calculation of the correlation potential and Schrödinger equation solution proceeds in the same way as in a standard atomic superposition calculation. Hereby, the effect of the charge transfer between $\mathrm{Cu}$ and $\mathrm{Fe}$ atoms (particle and host) is taken into account. In the process of searching $R$ we do not apply the three-step procedure explained at the beginning of this section. Instead, $\Delta V_{3}$ and $R$ are chosen to get the best possible agreement in the positron potential and positron density behavior between VASP and ATSUP calculations for a given particle and supercell size. There are two reasons for that: First, the supercells are rather small and due to the periodic boundary condition used the positron reflects a periodic array of particles rather than an isolated one. Second, assigning the bulk positron affinity to very small $\mathrm{Cu}$ particles (a few $\mathrm{Cu}$ atoms) in Fe can be problematic ${ }^{\dagger}$.

In total, we examined $1,2,3,4$, and $5 \mathrm{Cu}$ atom particles in the 54 atom supercell and 1, 5, 9, and $15 \mathrm{Cu}$ atom particles in the 128 atom supercell. For example, in Fig. 1a the positron potential for the 128 atom supercell containing a $15 \mathrm{Cu}$ atom particle is shown in a cubic direction going through the particle center. We plot three curves there. The first one is for the potential obtained using the VASP (referred hereafter to as the "V-curve"), the second one was determined using the ATSUP method with the shift applied as explained above with $R=1.6 \AA$ and $\Delta V_{3}=3.1 \mathrm{eV}$ ("A-curve"), and the third one was obtained using the ATSUP method without applying any shift for the positron potential

\footnotetext{
$\dagger$ Further calculations are in progress in order to test what the minimum size of $\mathrm{Cu}$ particles is where the positron affinity can be considered to have the same value as in bulk (bcc) Cu.
} 

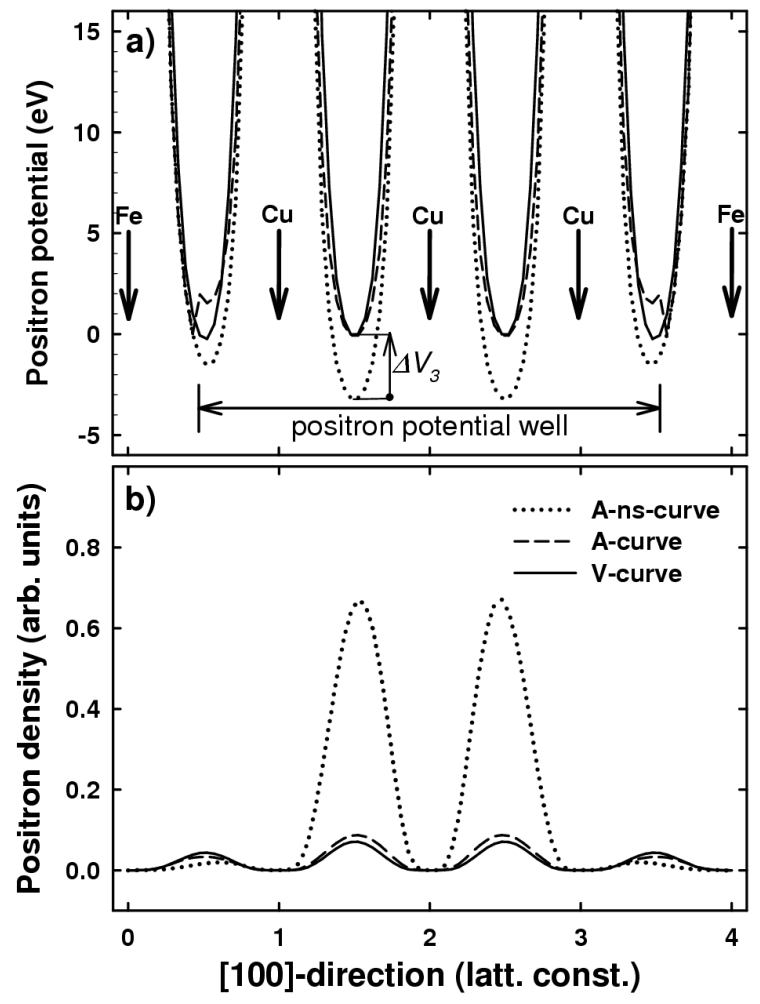

Fig. 1. The positron potential (a) and positron density (b) plotted in the [100] direction going through the center of the $15 \mathrm{Cu}$ atom particle embedded in the 128 atom supercell. See the text for the description of three curves (the scale is the same for all curves in either figure). The vertical arrows mark positions of $\mathrm{Fe}$ and $\mathrm{Cu}$ atoms. The extent of the positron potential well and $\Delta V_{3}$ shift are also indicated.

("A-ns-curve"). One can see that the A-curve approaches the V-curve rather well whereas the A-ns-curve deviates considerably from both $\mathrm{V}$ - and A-curves, which is due to the incorrect reference level of the positron potential (at the particle) in standard ATSUP calculations. Figure $1 \mathrm{~b}$ shows the behavior of the positron density in the same direction as in Fig. 1a for all three cases described above (referred to in the same way). Again the V-curve and A-curve agree very well, but the A-ns-curve clearly demonstrates that the attraction of positrons to the $\mathrm{Cu}$ particle is overestimated. A similar behavior is found for all other examined cases (with constant $R=1.6 \AA$ ). In conclusion, for all investigated particles and supercell sizes $R=1.6 \AA$ results in the acceptable behavior of the positron potential and density and this value is, therefore, chosen for our model calculations. 


\section{Results and discussion}

Having the values of model parameters we first inspect what the minimum size of a $\mathrm{Cu}$ particle is in which positrons can be trapped. For this purpose we employ the 1458 atom supercell $(9 \times 9 \times 9$ Fe bcc cells $)$ and examine the 9 and 15 $\mathrm{Cu}$ atom particles. These particles correspond, respectively, to atoms up to the first and second nearest neighbor shell of the central atom of the supercell used. The three-step procedure from [5] was employed, as explained at the beginning of the previous section. While the $9 \mathrm{Cu}$ atom particle does not represent a positron trap, the $15 \mathrm{Cu}$ atom particle exhibits clearly the positron localization at the particle though the corresponding positron binding energy to this particle is $0.05 \mathrm{eV}$ only. We may thus conclude that positron trapping occurs at particles starting from about $10-15 \mathrm{Cu}$ atoms. On the other hand, the well-known formula that estimates the minimum radius of particles that may trap positrons [3]

$$
r[\AA]=3.1 / \sqrt{\Delta A_{+}[\mathrm{eV}]}
$$

results in $r=3.7 \AA$ in our case. The $15 \mathrm{Cu}$ atom particle has an effective radius of $3.5 \AA$, which corresponds very well to the given estimate. This further justifies the validity of our model approach.

We now proceed with larger $\mathrm{Cu}$ particles. In our first study [15] devoted to the $\mathrm{Fe}-\mathrm{Cu}$ system we used another set of parameters $\left(R=2.9 \AA, \Delta A_{+}=1.0 \mathrm{eV}\right)$ derived from an analogy with the $\mathrm{Al}-\mathrm{Cu}$ system studied in [5]. In the present work the values of model parameters are justified on the basis of ab initio calculations and we present refined results of calculations of positron characteristics. We concentrate on the dependence of the $W$-parameter (or wing-parameter) on the precipitate size. The $W$-parameter characterizes PA with core electrons and is especially important when coincidence Doppler broadening (CDB) experiments are considered.

The range of momenta $(p)$ used for the calculation of the $W$-parameter was $15 \times 10^{-3} m_{\mathrm{e}} c<|p|<25 \times 10^{-3} m_{\mathrm{e}} c$. In this range the core electron contribution to the momentum distribution of annihilation photons (MDAP) dominates. The GC approach [12] for electron-positron correlations was used again. The calculations of the core electron contribution to the MDAP were carried using the computational scheme described in $[16]^{\ddagger}$. Furthermore, we employed the 2662 atom supercell $(11 \times 11 \times 11 \mathrm{Fe}$ bcc cells $) . \mathrm{Cu}$ particles were located in the center of the supercell occupying an increasing number of nearest neighbor shells of the central atom. Thus, we considered 15, 27, 51, 59, 65, and $89 \mathrm{Cu}$ atom particles.

The results of the $W$-parameter calculations are presented in Fig. 2. One can see a gradual saturation of the $W$-parameter with the increasing number of $\mathrm{Cu}$ atoms in particles to the value corresponding to $\mathrm{Cu}$ calculated also using the

\footnotetext{
${ }^{\ddagger}$ The number of $3 d$-electrons of Fe and $\mathrm{Cu}$ contributing to the MDAP was reduced to 3 and 4 , respectively, in order to reproduce the experimental CDB spectra of pure (defect free) Fe and $\mathrm{Cu}$ (see [17] for details).
} 


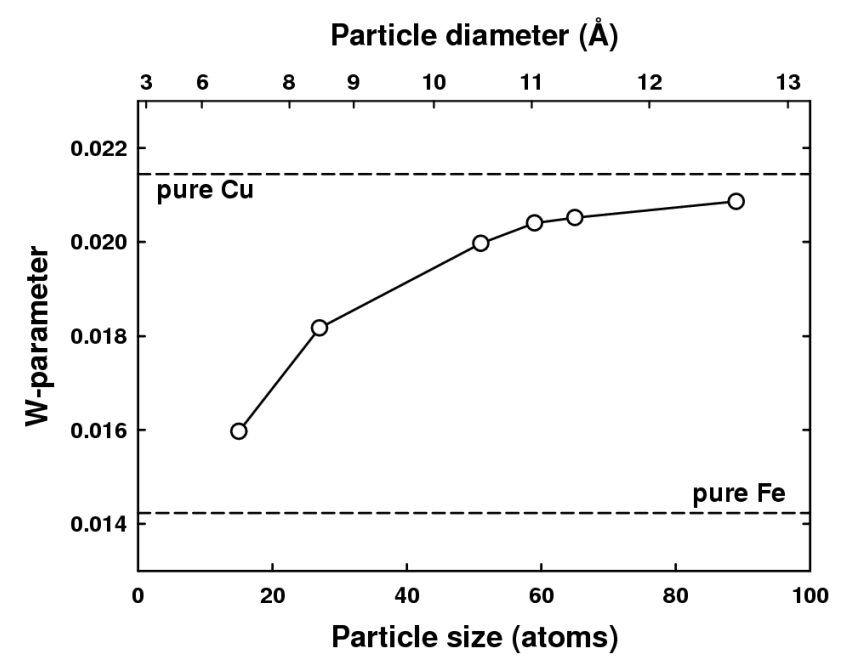

Fig. 2. The dependence of the $W$-parameter on the size of $\mathrm{Cu}$ particles embedded in the Fe matrix (the full line connecting calculated points is a guide to eyes only). Values of $W$ for pure (defect free) $\mathrm{Fe}$ and $\mathrm{Cu}$ are also specified.

ATSUP method (that reproduces well the experimental value). From Fig. 2 one can deduce an important piece of information that PA methods can be used to "measure" the size of $\mathrm{Cu}$ particles in the range from $\sim 7$ to $\sim 13 \AA$ and PA can be used to monitor early precipitation stages of $\mathrm{Cu}$ in $\mathrm{Fe}$ [15].

The computational method examined above can be also employed in large-scale positron simulations [17] performed for atomistic model samples of nanostructured materials. Nowadays, realistic model samples of nanostructures can be obtained using molecular dynamics and/or Monte Carlo methods [18]. In [15] we used the results of Monte Carlo simulations of the annealing process in the $\mathrm{Fe}-\mathrm{Cu}$ system to estimate the specific trapping rate of $\mathrm{Cu}$ precipitates in $\mathrm{Fe}$, which can be very useful when determining the concentration of $\mathrm{Cu}$ precipitates in real samples. Currently, new experimental data are being evaluated and will be coupled to positron simulations. The results will be published elsewhere [19].

Finally, it should be pointed out that the validity and accuracy of the model approach applied here to the $\mathrm{Fe}-\mathrm{Cu}$ system should be further tested by examining other systems containing embedded nanoparticles and by careful comparison with $a b$ initio calculations and appropriate experiments. In this context it is useful to mention the work of Nagai et al. [20] who investigated Guinier-Preston (GP) zones in the $\mathrm{Al}-\mathrm{Cu}$ system using coincidence Doppler broadening and lifetime spectroscopy. They did not observed positrons trapped at ideal (vacancy free) GP1 and GP2 zones in contrast to predictions presented in [5]. However, positron trapping at GP zones may be observable at low temperatures only because the positron binding energy to GP zones was roughly estimated to be as low as $0.3 \mathrm{eV}$ [5]. This effect can be also expected for small $\mathrm{Cu}$ particles in $\mathrm{Fe}$. 


\section{Acknowledgment}

The cooperation with A. Almazouzi, F. Bečvář, J. Č́ízek, C. Domain, L. Malerba, O. Melikhova, I. Procházka, S. Van Petegem, and K. Verheyen is gratefully acknowledged. We are indebted to O.K. Andersen and O. Jepsen for providing us with their LMTO code. We are also grateful to M.J. Puska and T. Korhonen for permitting us to use their LMTO positron and ATSUP codes, the latter one serving as a basis for further developments. This work has been funded within the 6th Framework Program under the Integrated Project PERFECT (FI6O-CT-2003-508840).

\section{References}

[1] V.L. Sedov, V.A. Teimurazova, K. Berndt, Phys. Lett. A 33, 319 (1970).

[2] M.J. Stott, P. Kubica, Phys. Rev. B 11, 1 (1975).

[3] M.J. Puska, P. Lanki, R.M. Nieminen, J. Phys., Condens. Matter 1, 6081 (1989).

[4] R. Saniz, B. Barbiellini, A. Denison, Phys. Rev. B 65, 245310 (2002).

[5] J. Kuriplach, F. Bečváŕ, J. Č́ízek, I. Procházka, Mater. Sci. Forum 445-446, 132 (2004).

[6] M.J. Puska, R.M. Nieminen, J. Phys. F, Metal Phys. 13, 333 (1983); A.P. Seitsonen, M.J. Puska, R.M. Nieminen, Phys. Rev. B 51, 14057 (1995).

[7] A. Bharathi, B. Chakraborty, J. Phys. F, Metal Phys. 18, 363 (1988).

[8] Y. Nagai, M. Hasegawa, Z. Tang, A. Hempel, K. Yubuta, T. Shimamura, Y. Kawazoe, A. Kawai, F. Kano, Phys. Rev. B 61, 6574 (2000).

[9] See, e.g., G.R. Odette, G.E. Lucas, J. Miner. Met. Mater. Soc. 53, 18 (2001); L. Malerba, E. van Walle, C. Domain, S. Jumel, J.-C. van Duysen, in: Proc. 10th Intern. Conf. on Nuclear Engineering (ICONE10), The American Society of Mechanical Engineers, New York 2002, paper no. 22260.

[10] For a recent review, see O.K. Andersen, O. Jepsen, M. Šob, in: Electronic Band Structure and its Applications, Ed. M. Yussouff, Springer Verlag, Heidelberg 1987, p. 1.

[11] D.W. Gidley, W.E. Frieze, Phys. Rev. Lett. 60, 1193 (1988); M. Jibaly, A. Weiss, A.R. Koymen, D. Mehl, L. Stiborek, C. Lei, Phys. Rev. B 44, 12166 (1991).

[12] B. Barbiellini, M.J. Puska, T. Korhonen, A. Harju, T. Torsti, R.M. Nieminen, Phys. Rev. B 53, 16201 (1996).

[13] G. Kresse, J. Hafner, Phys. Rev. B 47, 558 (1993); ibid. 49, 14251 (1994); G. Kresse, J. Furthmüller, Comput. Mat. Sci. 6, 15 (1996); G. Kresse, J. Furthmüller, Phys. Rev. B 54, 11169 (1996).

[14] P.E. Blöchl, Phys. Rev. B 50, 17953 (1994); G. Kresse, D. Joubert, Phys. Rev. $B$ 59, 1758 (1999).

[15] J. Kuriplach, S. Van Petegem, K. Verheyen, L. Malerba, A. Almazouzi, Ref. [19], p. 9.

[16] J. Kuriplach, A.L. Morales, C. Dauwe, D. Segers, M. Šob, Phys. Rev. B 58, 10475 (1998). 
[17] S. Van Petegem, J. Kuriplach, H. Van Swygenhoven, R. Meyer, C. Dauwe, D. Segers, Mater. Sci. Forum 445-446, 204 (2004).

[18] See, e.g., Modeling and Simulating Materials Nanoworld, in series Advances in Science and Technology, Vol. 44, Eds. P. Vincenzini, F. Zerbetto, Techna Group, Faenza 2004.

[19] K. Verheyen, A. Almazouzi, C. Domain, L. Malerba, J. Kuriplach, S. Van Petegem, to be published.

[20] Y. Nagai, M. Murayama, Z. Tang, T. Nonaka, K. Hono, M. Hasegawa, Acta Mater. 49, 913 (2001). 